\title{
Research on Rolling Bearing Fault Diagnosis with Adaptive Frequency Selection based on LabVIEW
}

\author{
Hongxin Zhang, Hao Zhou, Xianjiang Shi, Ju huang, Jixiang Sun and Lei Huang \\ School of Mechanical \& Power Engineering, Harbin University of Science \& \\ Technology, Heilongjiang, China \\ zhxlj2004@163.com
}

\begin{abstract}
In order to study the on-line fault monitoring and diagnosing for the rolling bearing this paper proposes a resonant demodulation measurement with an adaptive frequency selection based on LabVIEW. The wavelet packet function is used to decompose and reconstruct the measured vibration signal to extract the fault information accurately under the noise background. The kurtosis value of the signal within the range of all frequency bands is calculated and compared automatically to select a resonance frequency band containing the fault frequency. The fault frequency can be extracted by using the resonance demodulation and then the fault element can be identified. The experimental results show that the fault diagnosis result is the same as the fault simulation of the rolling bearing inner ring on the test bench.
\end{abstract}

Keywords: Fault diagnosis; Rolling bearing; Adaptive frequency selection; Resonance demodulation; LabVIEW

\section{Introduction}

Rolling bearing is widely used in rotating machinery, its failure will make equipments generate vibration and noise even damage. So it is the key to monitor and diagnose the fault of rolling bearings accurately for equipment management and predictive maintenance in modern enterprise. The study on on-line fault diagnosis of rolling bearing is very significant.

The common methods of fault diagnosis including temperature measurement, oil sample analysis and vibration test and so on. The vibration signal measurement and processing is widely used in rolling bearing fault diagnosis because of its simplification, intuition and reliability. The vibration test is to put sensors on bearing block or box in order to monitor vibration signal and then judge bearing conditions and failures by signal analyzing and processing. With the development of computer and communication technology, not only the vibration test technique is being developed rapidly but also some signal processing methods make bearing fault diagnosis technology more intelligent and multivariate, such as wavelet transform, neural network, genetic algorithm and so on ${ }^{[1-2]}$.

Some researches on rolling bearing fault diagnosis have been sophisticated. For example, acoustic emission signal is extracted by wavelet packet analysis to get energy characteristics which can be processed using the probabilistic neural network ${ }^{[3]}$. Another efficient method is to extract pulse signal by morphological gradient to get rolling bearing fault feature ${ }^{[4]}$. Recently the resonance demodulation is widely applied in fault diagnosis owing to its 
practicability. Some researchers use this method to extract the fault characteristic frequency directly based on LabVIEW ${ }^{[5]}$ or decompose and reconstruct vibration signal with wavelet packet $^{[6]}$. But how to find the fault characteristic frequency automatically and accurately under the noise is still difficult to realize, especially in the condition of on-line fault diagnosis. So this paper proposes a method of adaptive frequency selection based on LabVIEW which can select resonance frequency band automatically by analysis the distribution rule of the kurtosis value of signals from different frequency bands. In this research the resonance demodulation can be used by combining with the wavelet packet function.

\section{Rolling Bearing Fault Simulation and Diagnosis System}

The Rolling bearing fault simulation and diagnosis system includes three parts, they are rolling bearing fault simulation test bench, vibration signal detection circuit, fault diagnosis program based on LabVIEW.

Rolling bearing fault simulation test bench consists of three-phase asynchronous motor, flexible couplings, spindle, fault bearing, split housings and dc generator. The spindle is connected with motor's output shaft by flexible coupling and the other end is connected with dc generator by flexible coupling. There is an interference fit between shaft and inner ring and another clearance fit between outer ring and split housing. The test bed can simulate some damaging faults on outer ring, inner ring, ball and cage, such as pitting corrosion, wear and peeling.

The vibration signal detection circuit includes: a piezoelectric acceleration sensor (TS1100), a charge amplifier (TS5862), a band-pass filter (TS3795), a NI data acquisition card (DAQ - PCI - 6251) and a computer. The piezoelectric acceleration sensor is fixed on the split housing, the measured vibration signal is amplified by charge amplifier and filtered by band-pass filter and then converted to digital signal by NI DAQ.

Fault diagnosis program based on LabVIEW has the function of date acquisition and vibration analysis and processing. Rolling bearing fault simulation and diagnosis system is showed in Figure 1.

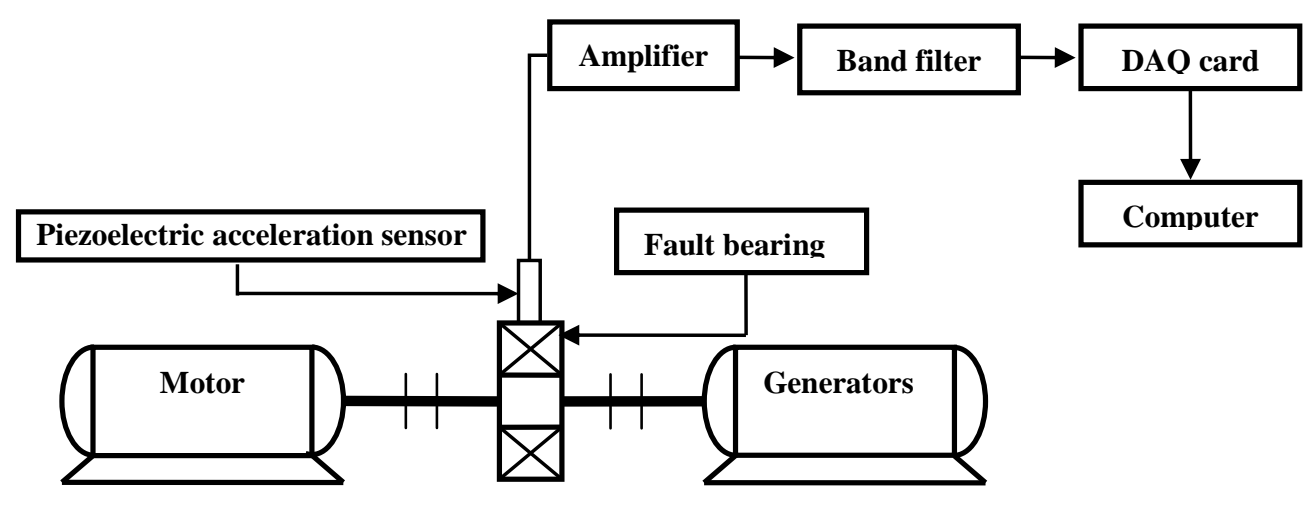

Figure 1. Rolling bearing fault simulation and diagnosis system 


\section{Fault Diagnosis Method of Adaptive Frequency Selection}

\subsection{Function modules based on LabVIEW}

Five modules are developed with LabVIEW software based on the idea of modularization. They are Data acquisition module, data reading and management module, fault characteristic frequency calculation module, data processing and analysis module and fault diagnosis module respectively. The five function modules work in parallel to analyze data and gain fault results. The LabVIEW function modules are showed in Figure 2.

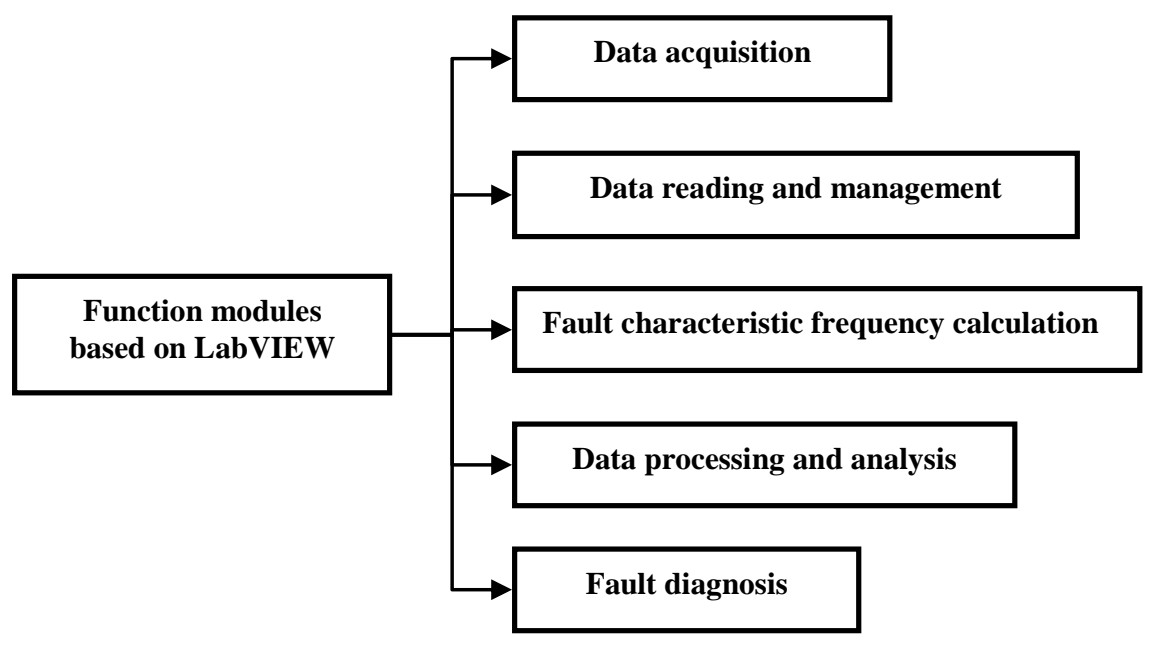

Figure 2. LabVIEW function modules

In the data acquisition module the parameters like sampling number, sampling rate and sampling mode can be set up conveniently, vibration signal curves and some parameters like effective value, peak value can be all showed in time domain, in the meanwhile, the amplitude spectrum can be displayed in frequency domain .

The data reading and management module can save the data and playback data in addition to read data on-line.

The fault characteristic frequency calculation module can calculate the fault characteristic frequency of damaging failure of single point in the light of parameters of bearing inner and outer ring, ball and cage size and spindle speed, etc. If the bearing outer ring is fixed and the inner ring can rotate, the following formula can be used to calculate fault characteristic frequency.

Inner ring fault frequency:

$$
f_{i c}=\frac{1}{2} N\left(1+\frac{d}{D} \cos \alpha\right) f_{r}
$$

Outer ring fault frequency:

$$
f_{o c}=\frac{1}{2} N\left(1-\frac{d}{D} \cos \alpha\right) f_{r}
$$


Roller elements fault frequency:

$$
f_{b c}=\frac{D}{2 d}\left[1-\left(\frac{d}{D} \cos \alpha\right)^{2}\right] f_{r}
$$

Cage fault frequency:

$$
f_{c e}=\frac{1}{2}\left(1-\frac{d}{D} \cos \alpha\right) f_{r}
$$

In the formula $f r$ is the spindle speed, $N$ is the roller number, $d$ is roller's diameter, $D$ is bearing diameter, $\alpha$ is bearing pressure angle.

In the data processing and analysis module the signal can be decomposed and reconstructed by the wavelet packet function and then be carried on the analysis of the adaptive frequency selection and the resonance demodulation.

Fault diagnosis module can compare the measured fault characteristic frequency with the calculated fault characteristic frequency. When the deviation is less than a constraint value, there will be a fault conclusion and a warning signal displayed.

\subsection{Principle of adaptive frequency selection fault diagnosis}

Bearing's vibration is caused by stiffness changes on the basis of bearing's structural characteristics, and alternating excitation force which is produced by Surface roughness, shape error and assembly error. Especially the damaging fault on the surface of bearings can cause impact vibration. In this paper the fault diagnosis is mainly aimed at impact vibration. The frequency distribution of the impact vibration is complicated. On the one hand the contact between damaging points and the bearing elements' surface will generate low-frequency strike on the other hand the impact can make some highfrequency components to generate resonance. That is, the high- frequency components will be modulated by low-frequency component and there will be some resonance frequency bands in the frequency domain. Low fault frequency cannot be extracted directly because of the low-frequency noise. So it is the key to determine a good band among several resonance frequency bands.

The principle of adaptive frequency selection is to decompose and reconstruct the original vibration signal using wavelet packet [7] and take statistical analysis to each frequency in amplitude spectrum. A maximum amplitude frequency is used as a bound condition to create an array which is indexed by the maximum kurtosis. Then the indexed frequency corresponding to the maximum amplitude is set to the filter's central frequency to carry on resonance demodulation and get the fault diagnosis result finally. The flow chart of resonance demodulation based on adaptive frequency selection is showed in Figure 3. 


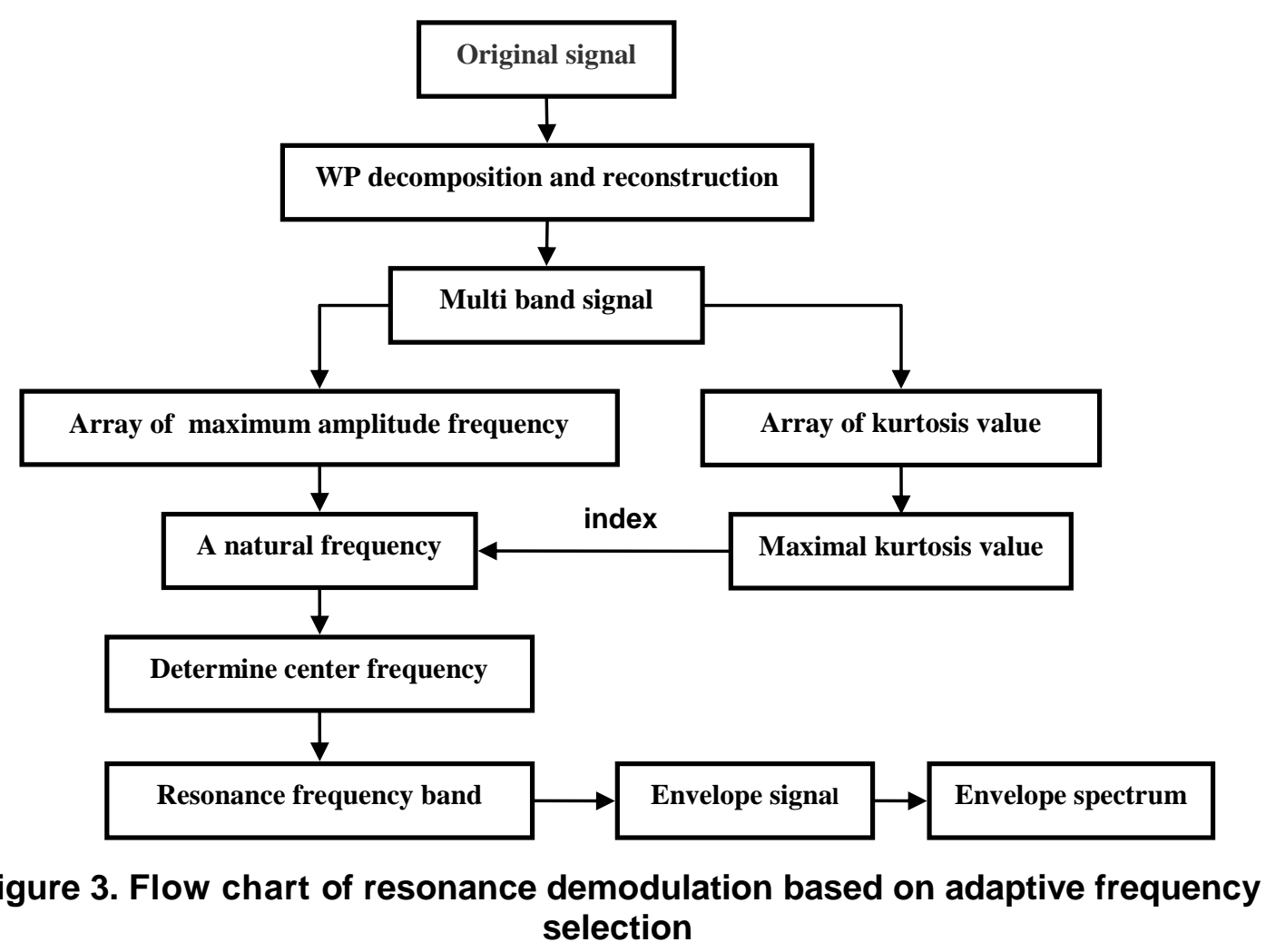

As an important statistical estimator the kurtosis coefficient (fourth order center distance) can be used to represent the steep degree of peaks of the sample graphics. The formula is

$$
K=\frac{1}{N} \sum_{i=1}^{N}\left[\frac{x_{i}-\mu}{\sigma}\right]^{4}
$$

$\mu$ is the sample mean, $\sigma$ is the sample variance. As above mentioned after wavelet packet decomposition and reconstruction for original signal, the kurtosis values of each frequency band signal can be calculated and the maximum kurtosis value is correspond to the resonance frequency band in which the fault information is the most distinguishable. It will get clear effect to run resonance demodulation to the frequency band. The frequency corresponding to the maximum amplitude on the frequency band is the natural vibration frequency, which is regarded as a band-pass filter's center frequency.

The Hilbert transform is used as resonance demodulation to get low frequency envelope signal from the amplitude modulation wave and extract the bearing fault characteristic frequency by spectrum analysis [8]. The principle of Hilbert transform is as follows,

$x(t)$ is a real time domain signal, the Hilbert transform formula is 


$$
h(t)=\frac{1}{\pi} \int_{-\infty}^{+\infty} \frac{x(\tau)}{t-\tau} d \tau=x(t) * \frac{1}{\pi t}
$$

A new analytic signal is composed of $x(t)$ and $h(t)$

$$
z(t)=x(t)+j h(t)=a(t) e^{j \varphi t}
$$

$z(t)$ is the amplitude analytic signal of $x(t)$.

\section{The Fault Diagnosis Experiment and Analysis}

In order to verify the accuracy of rolling bearing fault simulation and diagnosis system, an experiment on a deep groove ball bearing with peeling fault on inner ring has been done. The pitch diameter $\mathrm{D}$ of the bearing is $58.56 \mathrm{~mm}$, the roll diameter $d$ is $13.79 \mathrm{~mm}$, the roller number $\mathrm{N}$ is 8 , and the contact angle $\alpha$ is $0^{\circ}$. The failure bearing and the fault position are as shown in Figure 4.

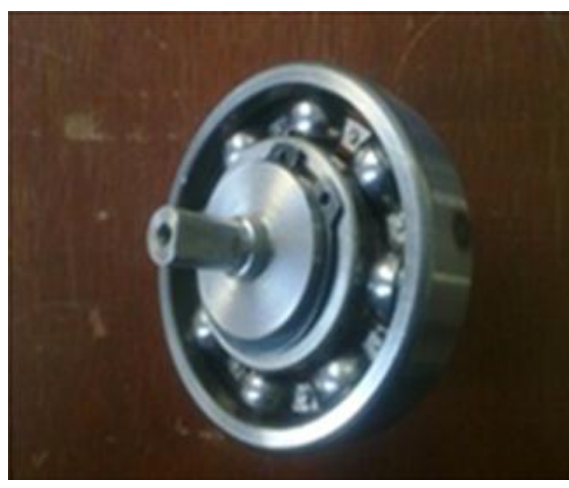

(a) Faulty bearing

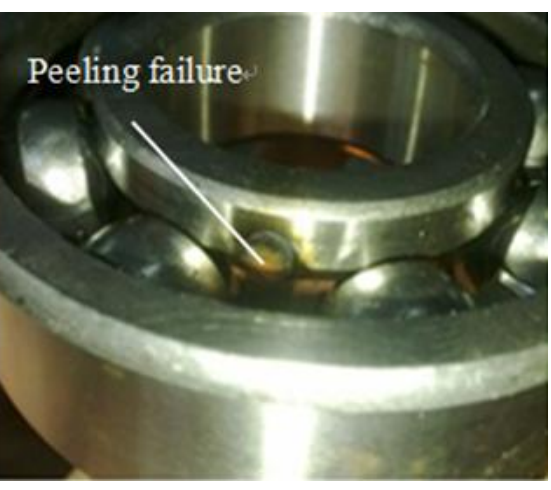

(b) Inner ring fault

Figure 4. Fault bearing and peeling failure of inner ring

The motor speed $\mathrm{n}$ is set to $1450 \mathrm{r} / \mathrm{min}$, the data acquisition sampling rate $f s$ is $1024 \mathrm{~Hz}$, and the sampling number is 1024. The fault characteristic frequency calculation module automatically calculates the characteristic frequency. The calculating results are that the failure frequencies of inner ring, external ring, rolling element and bearing support are $119.4 \mathrm{~Hz}, 73.9 \mathrm{~Hz}, 48.46 \mathrm{~Hz}$ and $9.237 \mathrm{~Hz}$ respectively.

The original vibration signal waveform and its amplitude spectrum are as shown in Figure 5, we can see the obvious impact in the signal waveform. 


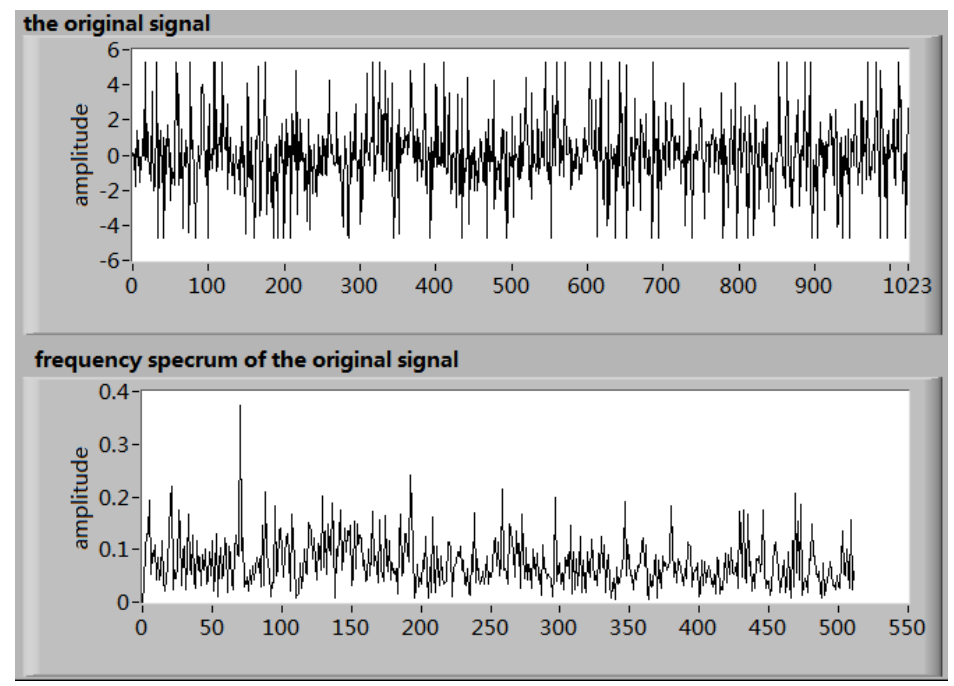

Figure 5. Original signal waveform and frequency spectrum

The original signal is divided into four time domain signals with different frequencies after four-layer wavelet packet decomposition showed in figure 6 . According to the frequency from the high to low the signals are $\mathrm{d} 1, \mathrm{~d} 2, \mathrm{~d} 3$ and $\mathrm{d} 4$ respectively, the calculated kurtosis values of four-layer signals are 3.974、3.360、3.943、3.542 respectively, the kurtosis value of the first layer is the maximum by comparing. The frequency band of the first layer signal is analyzed by the resonance demodulation. The maximum frequency $119 \mathrm{~Hz}$ can be seen in amplitude spectrum shown in Figure 7, which is equal to the calculated inner ring fault frequency $119.4 \mathrm{~Hz}$. Meanwhile the diagnosis result given by LabVIEW program is "Inner ring fault" which is the same as the simulated fault on the rolling bearing fault simulation test bench.

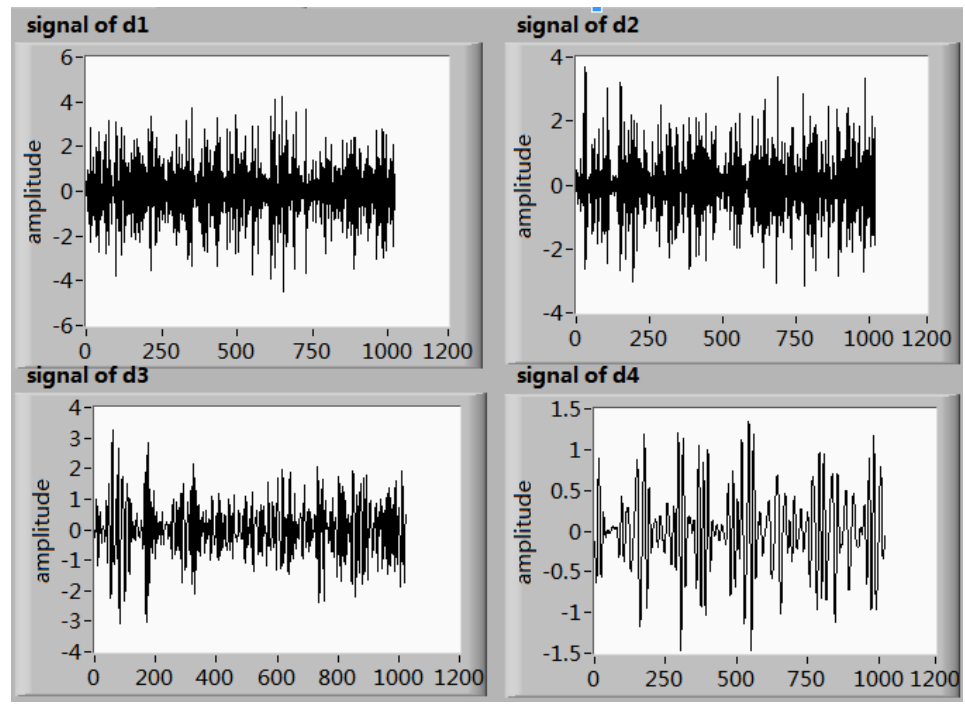

Figure 6. The wavelet packet transform signal 


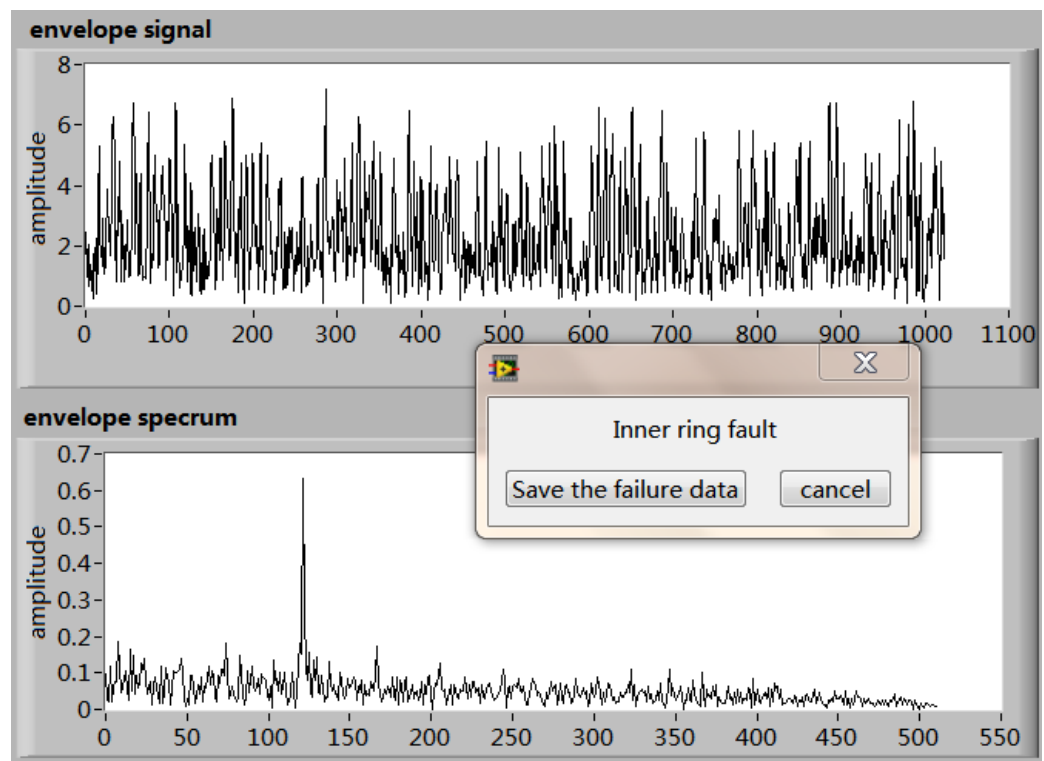

Figure 7. Envelope signal and envelope spectrum

\section{Conclusion}

A rolling bearing fault simulation and diagnosis system is built based on the LabVIEW software development platform. The system consists of fault simulation test bench, vibration signal detection circuit and fault diagnosis process. The adaptive frequency selection is implemented through the wavelet packet decomposition and reconstruction and the kurtosis value calculation. The experimental results show that the demodulated frequency value is in conformity with the calculated fault frequency and the fault diagnosis conclusion and the fault simulation of rolling bearing inner ring are the same. The consequence also indicates that the adaptive frequency selection is significant to find the fault frequency automatically under the noise in the on-line fault diagnosis of rolling bearing.

\section{Acknowledgements}

This research is supported by National Natural Science Foundation of China (51275136) and Natural Science Foundation of Heilongjiang Province of China (F201117).

\section{References}

[1] P. K. KanKar, C. S. Sharma and S. P. Harsha, "Neurocomputing”, vol. 110, (2013), pp. 9-17.

[2] R. B. Randall and J. Antoni, Mechanical Systems and Signal Processing, vol. 25, no. 2, (2007), pp. 485-520.

[3] C. C. Chen, Journal of Henan Polytechnic University, vol. 30, no. 1, (2011), pp. 62-66.

[4] X. Q. Qiu and X. Zhang, Coal Mine Machinery, vol. 28, no. 6, (2007), pp. 6-8.

[5] X. Chen and W. X. Li, "Journal of WUT", vol. 29, no. 1, (2007), pp. 42-43.

[6] L. Y. Lv, X. F. Tian and Y. Y. Lou, Machinery \& Electronics, vol. 7, (2010), pp. 75-77.

[7] X. Zhou, L. G. Hou, C. L. Su, Y. L. Xiao and Y. Zhang, Process Automation Instrumentation, vol. 33, no. 4, (2012), pp. 1-4.

[8] F. Pan, S. R. Quin and L Bai, China Measurement Technology, vol. 33, no. 4, (2007), pp. 10-12. 